\title{
Ongoing Trials: What Should We Expect After ALLHAT?
}

\author{
Ji-Guang Wang, $M D^{*}$, Jan A. Staessen, $M D, P h D^{*}$, \\ and Anthony M. Heagerty, $M D^{\dagger}$
}

\begin{abstract}
Address
*Study Coordinating Center, Hypertension and Cardiovascular Rehabilitation Unit, Department of Molecular and Cardiovascular Research, University of Leuven, Leuven, Beigium.

tDepartment of Medicine, Manchester Royal Infirmary, Oxford Road, Manchester MI3 9WL, UK.

E-mail: tony.heagerty@man.ac.uk

Current Hypertension Reports 2003, 5:340-345

Current Science Inc. ISSN 1522-6417

Copyright $(0) 2003$ by Current Science Inc.

The publication of the Antihypertensive and Lipid-lowering Treatment to Prevent Heart Attack Trial (ALLHAT) results, when used in conjunction with a new meta-analysis, provides hypertension research with a watershed. Data demonstrate clearly that lowering blood pressure is the most important aspect of hypertension management, and that all agents are similarly effective. There is little sustainable evidence for pressure-independent advantages for any class of drug at this time. Therefore, by combining these findings with additional information on target levels of blood pressure and safety, plus the use of aspirin and statins, we can formulate health care policies that attack hypertension in a holistic risk-based way. The future will focus on gaining extra benefit for patients by testing the efficacy and superiority of drug combinations using novel agents that favorably influence intermediate markers such as arterial stiffness, and arresting the ever-increasing burden of cognitive impairment and dementia.
\end{abstract}

\section{Introduction}

Hypertension management has evolved against a background of accumulating evidence, which has gradually convinced even the harshest skeptic that strategies directed at lowering blood pressure are associated with reductions in mortality and morbidity. Indeed, the unfolding story has been a triumph of clinical research, as trial design has been refined and improved, and in spite of the need to conduct intervention studies for years, each new study has brought us important information on how to attack a disease that affects more than $20 \%$ of our adults and ravages the elderly when untreated.
It is less than 20 years since we have learned that $\beta$ blocker- or diuretic-based regimens not only lower blood pressure, but also have an impact on stroke [1]. Indeed, the majority of treatment trials designed to demonstrate effects on hypertension-associated outcomes originally focused on trying to improve on these original results and impact upon the far greater burden of coronary heart disease (CHD). The effects of hypertension outcomes on cerebrovascular disease appear to be immediate, whereas those on CHD appear more subtle and over a more protracted time course. Clues on to how to proceed are already available historically. The Veterans' study [2] convincingly showed that hypertension-associated heart failure could be abolished largely by a modest reduction in pressure. New trial designs targeted older and obviously more at-risk patients, and by using $\beta$-blockers or diuretics better outcomes were achieved for heart disease [3-5].

Three important developments followed. First, there is the increased awareness that the level of systolic blood pressure is as prognostically important as the diastolic reading. Furthermore, isolated systolic hypertension is the predominant form of hypertension as we age, and far from being an irreversible part of the aging process, this too can be lowered with drugs with favorable outcomes on stroke and heart disease. Second, new classes of drugs are being used as the basis of outcome trials as investigators test the claim that these drugs are superior to $\beta$-blockers and diuretics. Third, because of concerns about lowering blood pressure too much, a landmark study was performed and it demonstrated that lowering diastolic blood pressure below $90 \mathrm{~mm} \mathrm{Hg}$ did not result in increased mortality or morbidity [6]. However, how strict blood pressure control should be remains uncertain. Where are we now?

Drawing together the accumulating experience of past trials, more recent investigations have recruited elderly patients with either isolated systolic or combined systolic and diastolic hypertension. Also, to increase the outcome event rates, it has become customary to use patients with other cardiovascular risk factors such as diabetes, hypercholesterolemia, or previous stroke or heart disease. In addition, because of clear benefits from interventions, the placebo has long been discarded. Trials are comparing newer agents such as angiotensin-converting enzyme (ACE) inhibitors, calcium channel blockers, and $\alpha$-block- 
ers with so-called older agents such as $\beta$-blockers and diuretics with trial established records. The contention is that newer drugs might act hemodynamically more favorably, thereby improving outcome, or perhaps might possess intrinsic, pressure-independent properties that again would favor their use in hypertension. Much of the evidence for this latter position has emerged from animal research, which uses doses of agents in excess of what is possible in humans, or extrapolation to human studies of heart failure, where until recently ACE inhibitors were one of only two classes of drug established to prolong survival.

Therefore, the publication of the Heart Outcomes Prevention Evaluation (HOPE) study produced intense interest $[7 \cdot]$. Apparently, here was evidence that the addition of the ACE inhibitor ramipril to established treatment regimens in high-risk patients produced additional protection, which could not be ascribed to a fall in blood pressure. Indeed, the health gain from this maneuver was impressive. However, blood pressure data from a substudy of patients in the HOPE trial were published subsequently, and it is possible that pressure falls were greater than previously realized [8]. Furthermore, in the Non-InsulinDependent Diabetes, Hypertension, Microalbuminaria, or Proteinuria, Cardiovascular Events and Ramipril (DIABHYCAR) study, which was a randomized, prospective, doubleblind, placebo-controlled trial of low-dose ramipril in type II diabetic patients, ramipril reduced systolic pressure by $2.1 \mathrm{~mm} \mathrm{Hg}$ but did not significantly change outcome [9].

The case for a favorable pressure-independent effect on cardiovascular outcomes using agents that inhibit angiotension II was apparently strengthened by the Losartan Intervention For Endpoint Reduction in Hypertension (LIFE) study [10•]. This included 9193 patients randomized to receive losartan or atenolol in a double blind, parallel group design. Primary outcomes included nonfatal myocardial infarction, nonfatal stroke, and mortality due to myocardial infarction, stroke, sudden death, heart failure, or other cardiovascular causes Losartan significantly reduced relative risk in all patients and was even better in a subgroup of diabetics.

At this juncture, it is important to state that in largescale intervention trials involving thousands of patients, a mean difference of one or two $\mathrm{mm} \mathrm{Hg}$ pressures in groups before randomization or after target levels have been achieved might be extremely important in determining efficacy in terms of outcome. In other words, before invoking pressure-independent mechanisms to explain differences in mortality and morbidity, we need to look closely at what is achieved in terms of blood pressure control.

\section{Meta-analysis}

An attempt at examining this issue was undertaken recently [11]. A search was made of outcome trials that tested drugs to lower blood pressure in normotensive or hypertensive patients who did not have overt heart failure at enrollment. Other inclusion criteria were a randomized controlled design, publication in a peer-reviewed journal, inclusion of patients with hypertension assessment of blood pressure and cardiovascular events, follow-up of 2 years or longer, and sample size of 100 or more [5]. Nine studies were included involving 62,605 hypertensive patients who had been randomized to conventional therapy with diuretics or $\beta$-blockers or to initial treatment with newer agents such as calcium channel blockers, ACE inhibitors, or $\alpha$-blockers. Compared with conventional therapy, calcium channel blockers and ACE inhibitors offered similar overall cardiovascular protection, but calcium channel blockers provided more reduction in the risk of stroke and less reduction in the risk of myocardial infarction. The findings confirmed a previous meta-analysis [12] and the message seems clear: using this analytical approach, older and newer antihypertensive drugs confer similar protection.

Applying the meta-regression curve analysis technique to the HOPE and LIFE studies should provide an indication of whether small differences in achieved blood pressure could have influenced the outcome. The data from HOPE and LIFE fit well [13], and again the message is clear: it is the blood pressure control that is important in explaining the findings.

\section{ALLHAT}

The Antihypertensive and Lipid-lowering Treatment to Prevent Heart Attack Trial (ALLHAT) was designed to address prospectively whether the occurrence of fatal CHD or nonfatal myocardial infarction is lower for high-risk patients with hypertension treated with a calcium channel blocker (amlodipine), an ACE inhibitor (lisinopril), or an $\alpha$-blocker (doxazosin), each compared with diuretic treatment (chlorthalidone) [14*]. Secondary outcomes included all-cause mortality, stroke, and other cardiovascular disease events. In order to evaluate possible heterogeneity in cardiovascular disease effects of the various agents previously suggested by the meta-analysis approach, ALLHAT was designed with a large sample size (9000-15,000 patients per intervention arm) and long follow-up (4-8 years). Also such a large-scale investigation allowed the relative benefit of various agents in high-risk hypertensive subgroups such as older patients, black patients, and diabetics to be examined.

Patients were hypertensive men and women aged 55 years or older, with at least one additional risk factor for CHD. These included previous myocardial infarction or stroke, left ventricular hypertrophy, history of type 2 diabetes, current cigarette use, low high-density lipoprotein, or documentation of other atherosclerotic cardiovascular disease. Patients with heart failure were excluded.

The results are impressive: chlorthalidone was found to be superior to doxazosin and the $\alpha$-blocker arm was 
Table I. Observed odds ratios and odds ratios predicted by between-group differences in systolic pressure in recent trials of new versus old drugs

\begin{tabular}{|c|c|c|c|c|}
\hline Trial and events & $\begin{array}{l}\text { Change in systolic } \\
\text { blood pressure }\end{array}$ & Observed & Predicted* & $p^{\dagger}$ \\
\hline \multicolumn{5}{|l|}{ LIFE—all patients } \\
\hline Cardiovascular mortality & 1 & $0.87(0.72-1.05)$ & $0.90(0.78-1.05)$ & 0.75 \\
\hline Cardiovascular events & $i$ & $0.85(0.76-0.96)$ & $0.93(0.85-1.02)$ & 0.24 \\
\hline Stroke & 1 & $0.74(0.63-0.88)$ & $0.87(0.79-0.95)$ & 0.11 \\
\hline Myocardial infarction & $i$ & $1.05(0.86-1.28)$ & $0.93(0.85-1.02)$ & 0.28 \\
\hline \multicolumn{5}{|l|}{ LIFE-diabetic patients } \\
\hline Cardiovascular mortality & 3 & $0.62(0.4 \mid-0.92)$ & $0.86(0.76-0.99)$ & 0.12 \\
\hline Cardiovascular events & 3 & $0.73(0.57-0.95)$ & $0.84(0.77-0.91)$ & 0.34 \\
\hline Stroke & 3 & $0.78(0.54-1.13)$ & $0.78(0.71-0.85)$ & 0.99 \\
\hline Myocardial infarction & 3 & $0.81(0.54-1.22)$ & $0.85(0.78-0.93)$ & 0.82 \\
\hline \multicolumn{5}{|l|}{ ALLHAT/amlodipine } \\
\hline Cardiovascular mortality & -1.1 & $1.01(0.90-1.12)$ & $1.00(0.82-1.22)$ & 0.94 \\
\hline Cardiovascular events & -1.1 & $1.04(0.99-1.09)$ & $1.07(0.95-1.21)$ & 0.60 \\
\hline Stroke & -1.1 & $0.93(0.82-1.06)$ & $1.00(0.89-1.12)$ & 0.43 \\
\hline Myocardial infarction & -1.1 & $0.98(0.90-1.07)$ & $1.06(0.93-1.20)$ & 0.30 \\
\hline \multicolumn{5}{|l|}{ ALLLHAT/lisinopril-all patients } \\
\hline Cardiovascular mortality & -2.3 & $1.04(0.93-1.15)$ & $1.06(0.84-1.33)$ & 0.90 \\
\hline Cardiovascular events & -2.3 & $1.10(1.05-1.16)$ & $1.17(1.02-1.35)$ & 0.39 \\
\hline Stroke & -2.3 & $1.15(1.02-1.30)$ & $1.08(0.94-1.24)$ & 0.51 \\
\hline Myocardial infarction & -2.3 & $0.99(0.91-1.08)$ & $1.14(0.98-1.34)$ & 0.09 \\
\hline \multicolumn{5}{|l|}{ ALLHAT/lisinopril-patients > 65 years } \\
\hline Cardiovascular events & -3.0 & $1.13(1.06-1.20)$ & $1.24(1.06-1.45)$ & 0.27 \\
\hline Stroke & -3.0 & $1.13(0.98-1.30)$ & $1.14(0.98-1.32)$ & 0.93 \\
\hline Myocardial infarction & -3.0 & $1.01(0.91-1.12)$ & $1.20(1.01-1.43)$ & 0.08 \\
\hline \multicolumn{5}{|l|}{ ALLHAT/lisinopril-black patients (2736) } \\
\hline Cardiovascular events & -4.0 & $1.19(1.09-1.30)$ & $1.34(1 . \mid 2-1.61)$ & 0.23 \\
\hline Stroke & -4.0 & $1.40(1.17-1.68)$ & $1.23(1.03-1.47)$ & 0.31 \\
\hline Myocardial infarction & -4.0 & $1.10(0.94-1.28)$ & $1.29(1.05-1.59)$ & 0.20 \\
\hline
\end{tabular}

terminated (controversially) early [14•]. Mean follow-up was for 4.9 years, and the primary outcome of fatal CHD or nonfatal myocardial infarction occurred in 2956 patients with no difference between treatments. In addition, allcause mortality did not differ between groups. It is important to note that systolic pressures were significantly higher on amlodipine $(0.8 \mathrm{~mm} \mathrm{Hg})$ and lisinopril $(2 \mathrm{~mm}$ $\mathrm{Hg}$ ) compared with chlorthalidone; diastolic pressure was lower on amlodipine $(0.8 \mathrm{~mm} \mathrm{Hg})$; and lisinopril, amlodipine, and chlorthalidone achieved control of hypertension ( $<140 / 90 \mathrm{~mm} \mathrm{Hg}$ ) in $61 \%, 66 \%$, and $68 \%$ of patients, respectively. In terms of secondary outcomes, chlorthalidone was superior (perhaps predictably) in preventing heart failure, and chlorthalidone was better than lisimopril in preventing stroke, combined cardiovascular disease, and heart failure. As an important side issue, the safety concerns about long-acting calcium channel blockers were allayed by this study, and despite the widely held view that the metabolic effects of diuretics such as hypokalemia and glucose intolerance might adversely influence efficacy, this again was disproved. Finally, the substantial contribution of women and black patients to the cohort means the results can be extrapolated to the whole Western hypertensive community.

\section{ALLHAT and Meta-regression Analysis}

The observed versus predicted odds ratios for ALLHAT and LIFE are shown in Table 1. The LIFE comparisons have been published already [13]. Only one parameter attains significance in a subgroup comparison of the ALLHAT study. It is possible also to test the robustness of the metaregression technique by superimposing the ALLHAT and LIFE data on the meta-regression curves calculated before they were known. These are shown for fatal and nonfatal stroke in Figure 1 (before) and Figure 2 (with LIFE and ALLHAT). The message is clear once more: it is the level of blood pressure achieved that is responsible primarily for reported outcome, a point reinforced by Figure 3.

\section{The Holistic Approach to Hypertension}

The wealth of trial data now available allows us to make unequivocal statements about treating the hypertensive 


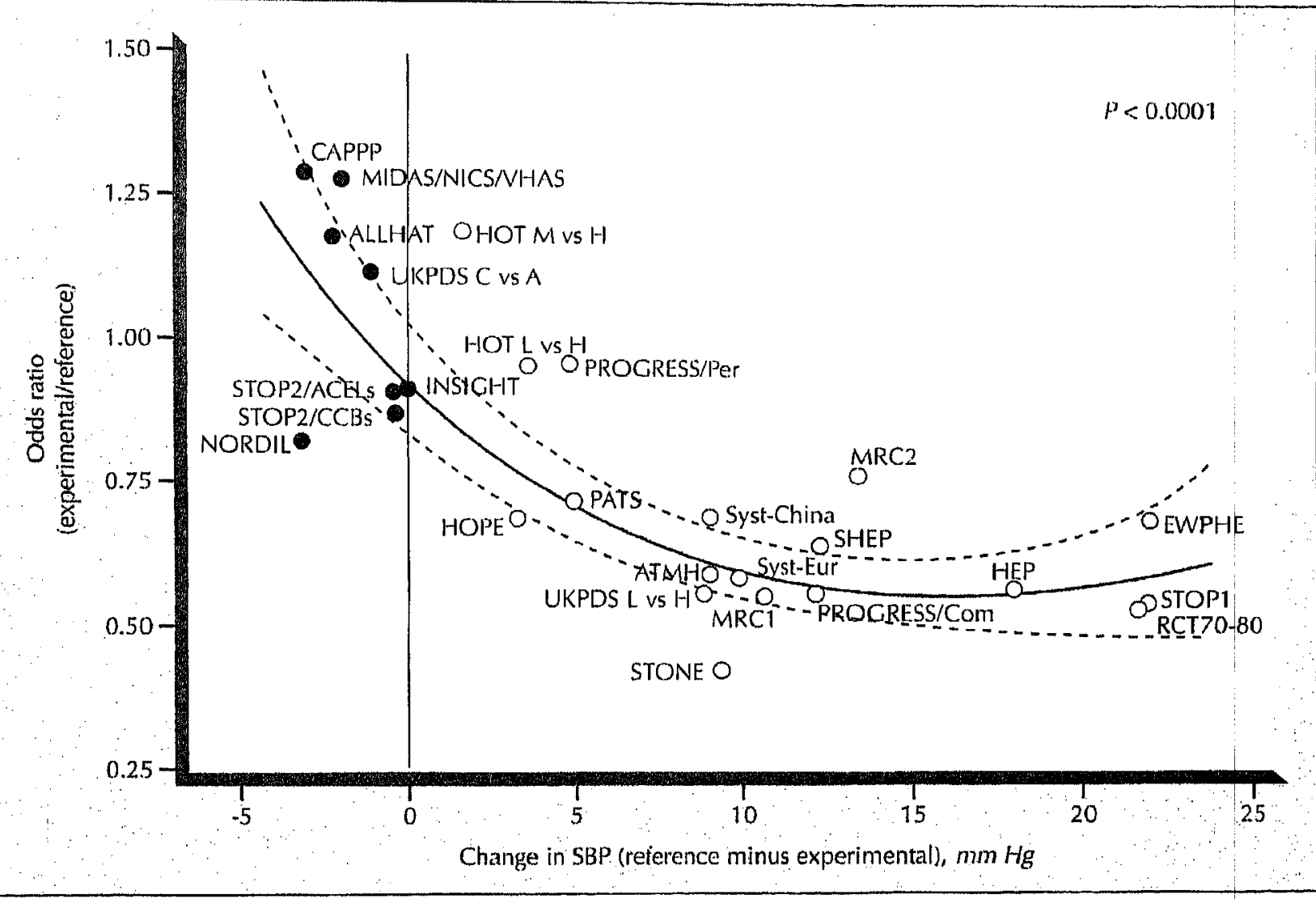

Figure 1. Relation between odds ratios for fatal and nonfatal stroke and corresponding differences in systolic blood pressure (SBP) in trials of old and new agents. (Data from Staessen et al. $[111$.)

patient. Survival is improved if control is tight ( $<140 / 90$ or preferably lower), and in patients over 50 years of age with evidence of vascular disease, aspirin improves outcome further as does the control of raised cholesterol; data from the Anglo Scandinavian Cardiovascular Outcome Trial (ASCOT) already reinforce this position. Our approach to hypertension in diabetics should be even more vigilant because in all the recent major cardiovascular outcome studies, these patients have received the greatest benefit.

\section{Choice of Agent}

In an age where health care costs continue to rise and in which many hypertensive patients fail to receive adequate treatment, it may make economic sense to use low-dose diuretics as first-line treatment unless there are contraindications or unacceptable side effects intervene. However, this remains to be established beyond doubt. No evidence of cost effectiveness was given in the ALLHAT study report. Patients given diuretics undergo blood tests periodically to check for metabolic and electrolyte disorders, and the incidence of diabetes, the treatment of which may have revenue consequences, is higher on diuretics. In addition, the availability of generic ACE inhibitors and calcium channel blockers will reduce the current treatment costs. However, other drug classes are as good and as safe, and two thirds of patients will need more than one agent to achieve good blood pressure control. The overwhelming public health benefit of treating hypertension should drive the case for identifying as many patients as possible to enter comprehensive management programs.

\section{Conclusions}

In terms of cardiovascular outcomes and class comparisons, the ASCOT study has yet to be completed but may give additional information on the use of aspirin and has already confirmed the usefulness of statins in risk reduction. More outcome data will emerge for angiotensin receptor blockers, but given what we have learned already, it is difficult to predict class superiority over pre-existing drugs. Studies will soon report on whether treatment should be extended to very old hypertensive patients: we should hope for and anticipate positive outcomes, and it is on the elderly population that we should concentrate our trial efforts. Inevitably the proportion of older people in acculturated societies will continue to grow and the burden 


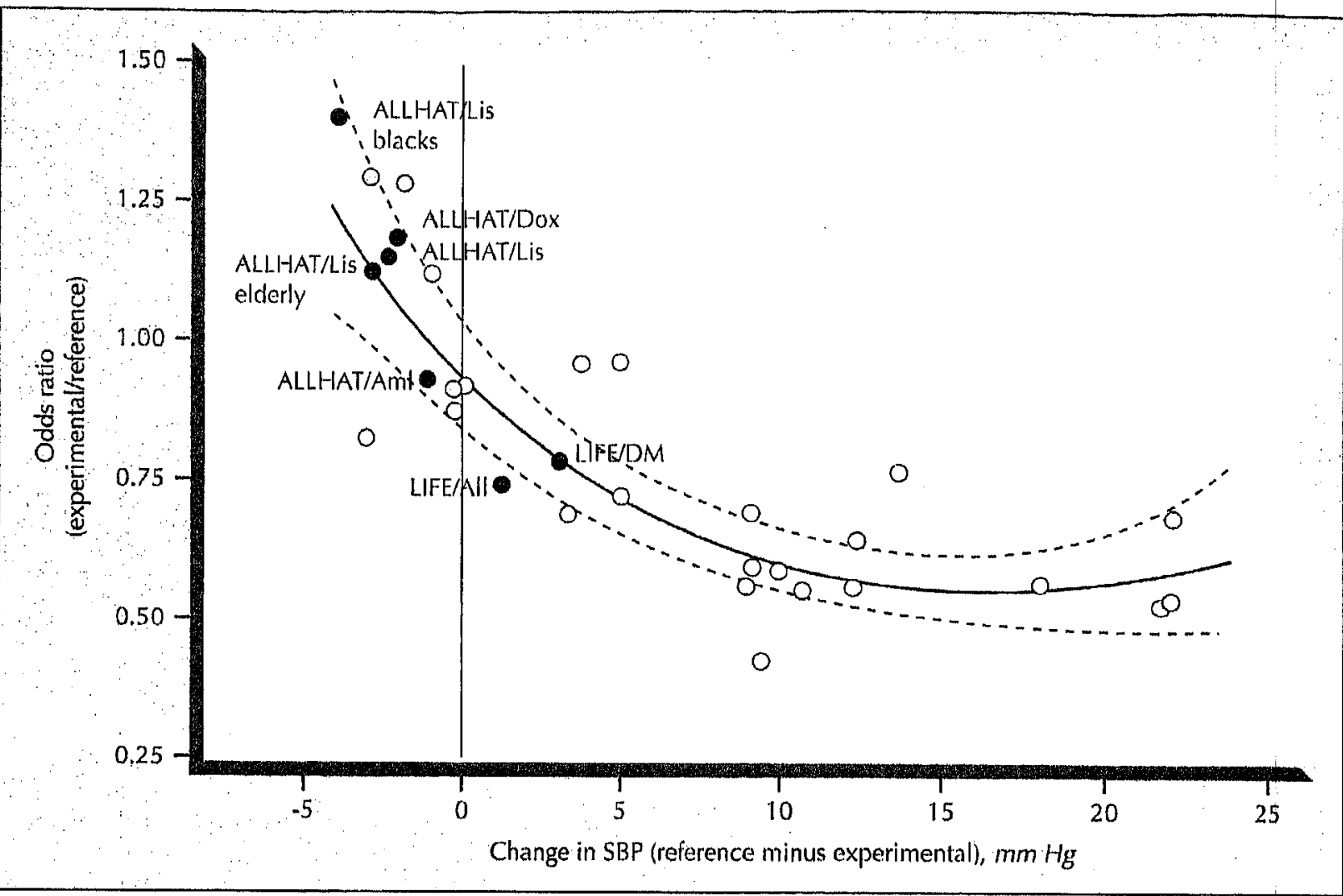

Figure 2. Relation between odds ratios for fatal and nonfatal stroke and corresponding differences in systolic blood pressure (SBP). ALLHAT and LIFE results were plotted superimposed on the meta-regression line.

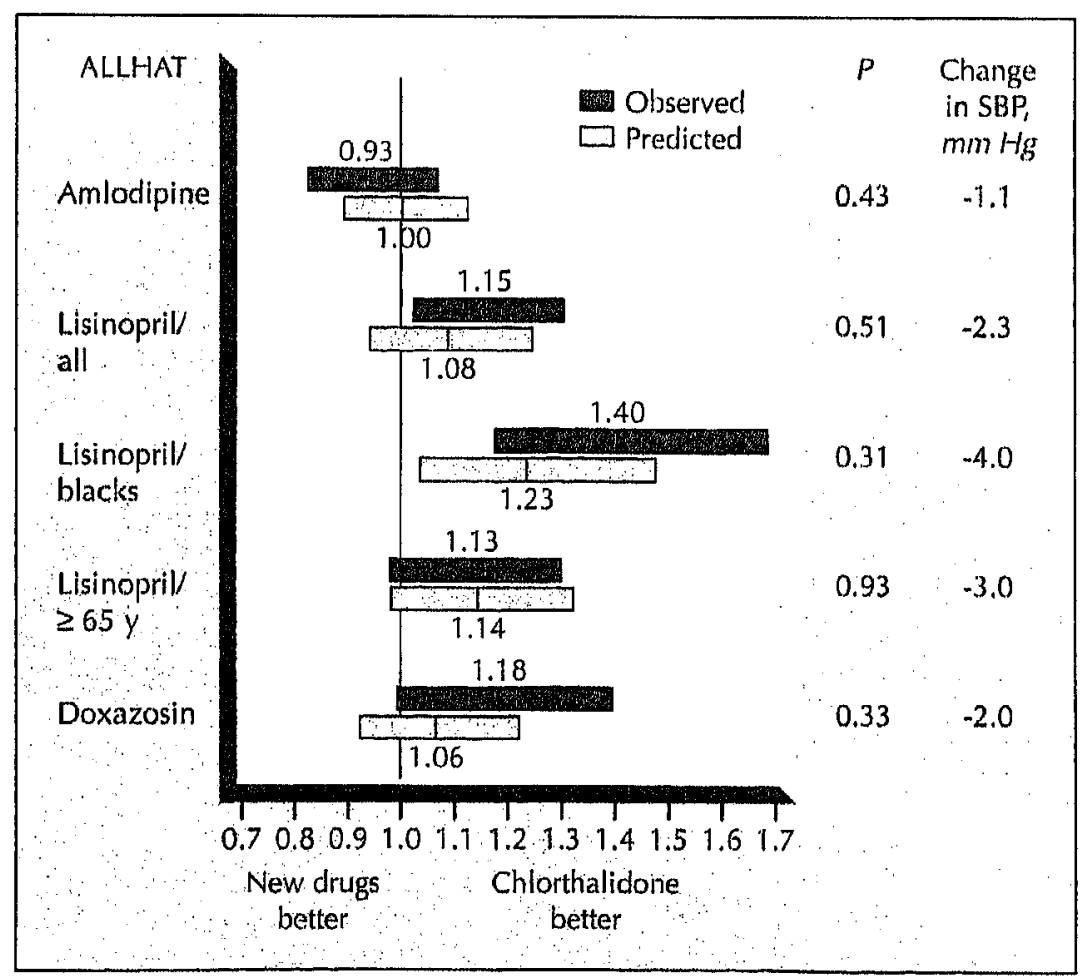

Figure 3. Observed and predicted odds ratios for stroke in the ALLLHAT study, predicted by meta-regression. SBP-systolic blodd pressure. (Data from Staessen ot al. $\mid 1$ 11.) 
of systolic hypertension will rise as a consequence. There is still a major challenge to better manage this form of disease. The expanding literature associating pulse pressure and arterial stiffness with cardiovascular risk places strategies that attempt to restore the integrity of large and medium-sized vascular tissue function high on the future therapeutic agenda. Similarly, a combination of hypertension control with new cytokine antagonists may retard atheroma development and regress plaque formation and rupture. In other words, the future must allow the development and testing of treatment strategies designed to add value to blood pressure control. The search for a single drug entity that will give benefit beyond blood pressure control is an obsolete concept given the multifactorial nature of hypertension, unless pharmacogenomics identifies patient populations who will respond favorably to a particular agent.

\section{References and Recommended Reading}

Papers of particular interest, published recently, have been highlighted as:

- Of importance

- Of major importance

1. Medical Research Council Working Party: MRC trial of trentment of mild hypertension: principal results. BMJ 1985. 291:97-104.

2. Veterans Administration Cooperative Study Group on Antihypertensive Agents: Effects of treatment on morbidity in hypertension II: results in patients with diastolic blood pressure averaging 90 through $114 \mathrm{~mm} \mathrm{Hg}$. IAMA 1979, 213:1143-1152.

3. SHEP Cooperative Research Croup: Prevention of stroke by antihypertensive drug treatment in older persons with isolated systolic hypertension. Final results of the Systolic Hypertension in the Elderly Program (SHEP). IAMA 1991, 265:3255-3264.

4. Dahlö $B$, Lindholm LH, Hansson $L$, et al.: Morbidity and mortality in the Swedish Trial in Old Patients with Hypertension. (STOP - Hypertension). Lancet 1991, 338:1281-1285.
5. MRC Working l'arty: Medical Research Council trial of treatment of hypertension in older adults: principal results. BMJ 1992, 304:405-412.

6. Hansson L, Zancheti A, Carruthers SG, el al.: Effects of intensive blood-pressure lowering and low-dose aspirin in patients with hypertension: principal results of the Hypertension Optimal Treatment (HOT) randomised trial. Lancet 1998, 351:1755-1762.

7. The Heart Outcomes Prevention Evaluation Study Investigators: Effects on an anglotensin-converting-enzyme inhibitor, ramipril, on cardiovascular events in high-risk patients. N Engl / Med 2000, 342:145-153.

The addition of the ACE inhibitor ramipril to established treatment regimens in high-risk patients produced additional protection, which could not be ascribed to a fall in blood pressure.

8. Svenson $P$, de Faire $U I$, Sleight $P$, et al: : Comparative effects of ramipril on ambulatory and office blood results: a HOPE substudy. Hyperterision 2001, 38:e28-e32.

9. The Non-Insulin-Dependent Diabetes, Hypertension, Microalbuminaria or Proteinuria, Cardiovascular Events and Ramipril (DIABHYCAR) Study: design, organisation and patient recruitment. Control Clin Trial 2000, 21:383-396.

10. Lindholm LH, Ibsen H, Dahlöf B, et al.: Cardiovascular morbidity and mortality in patients with diabetes in the Losartan Intervention For Endpoint reduction in hypertension study (LIFE); a randomised trial against atenolol. Lancet 2002, 359:1004-1010.

In this study, losartan significantly reduced relative risk in all patients and was even better in a subgroup of diabetics.

11. Staessen $1 \mathrm{~A}$, Wang Ji-Guang, Thijs L: Cardiovascular protection and blood pressure reduction: a meta-analysis. Lancet 2001, 358:1305-1315.

12. Collins R, MacMahon S: Blood pressure, antihypertensive drug treatment and the risks of stroke and of coronary heart disease. Br Med Bull 1994, 50:272-298.

13. Staessen J, Wang J-G, Birkenhager WH: Outcome beyond blood pressure control? Eur Heart / 2003, 24:504-514.

14. The ALLHAT Officers and Coordinators for the ALLIJAT Collaborative Research Group: Major outcomes in high-rlsk hypertensive patients randomised to angiotensin-coverting enzyme inhibitor or calcium channel bloder vs diuretic. The Antihypertensive and Lipid-Lowering Treatment to Prevent Heart Attack Trial (ALLHAT). JAMA 2002, 288:2981-2997. Chlorthalidone was found to be superior to doxazosin. In terms of secondary outcomes, chlorthalidone was superior in preventing heart failure, and was better than lisinopril in preventing stroke, combihed cardiovascular disease, and heart failure. 